PHYSICAL REVIEW C 71, 029903(E) (2005)

\title{
Publisher's Note: Fragment yield distribution and the influence of neutron composition and excitation energy in multifragmentation reactions
} [Phys. Rev. C 71, 024602 (2005)]

\author{
D. V. Shetty, A. S. Botvina, S. J. Yennello, G. A. Souliotis, E. Bell, and A. Keksis
}

(Received 4 February 2005; published 16 February 2005)

DOI: 10.1103/PhysRevC.71.029903(E)

PACS number(s): 25.70.Mn, 25.70.Pq, 26.50.+x, 99.10.Fg

This paper was published online on 2 February 2005 with an incorrect received date on the PDF file of the article. The received date should be 14 September 2004. The date has been corrected as of 4 February 2005. The received date is correct in the printed version of the journal. 